\title{
Expansion Dynamics after Laser-Induced Cavitation in Liquid Tin Microdroplets
}

\author{
Dmitry Kurilovich, ${ }^{1,2}$ Tiago de Faria Pinto, ${ }^{1,2}$ Francesco Torretti, ${ }^{1,2}$ Ruben Schupp, ${ }^{1}$ Joris Scheers, ${ }^{1,2}$ \\ Aneta S. Stodolna, ${ }^{1,2}$ Hanneke Gelderblom, ${ }^{3}$ Kjeld S.E. Eikema, ${ }^{1,2}$ Stefan Witte, ${ }^{1,2}$ Wim Ubachs, ${ }^{1,2}$ \\ Ronnie Hoekstra, ${ }^{1,4}$ and Oscar O. Versolato ${ }^{1, *}$ \\ ${ }^{1}$ Advanced Research Center for Nanolithography, Science Park 110, 1098 XG Amsterdam, Netherlands \\ ${ }^{2}$ Department of Physics and Astronomy, and LaserLaB, Vrije Universiteit Amsterdam, De Boelelaan 1081, 1081 \\ HV Amsterdam, Netherlands \\ ${ }^{3}$ Department of Applied Physics, Eindhoven University of Technology, P.O. Box 513, 5600 MB Eindhoven, \\ Netherlands \\ ${ }^{4}$ Zernike Institute for Advanced Materials, University of Groningen, Nijenborgh 4, 9747 AG Groningen, \\ Netherlands
}

(Received 18 May 2018; revised manuscript received 24 August 2018; published 2 November 2018)

\begin{abstract}
The cavitation-driven expansion dynamics of liquid tin microdroplets set in motion by the ablative impact of a 15-ps laser pulse is investigated. We combine high-resolution stroboscopic shadowgraphy with an intuitive fluid-dynamic model that includes the onset of fragmentation, and find good agreement between model and experimental data for two different droplet sizes over a wide range of laser pulse energies. The dependence of the initial expansion velocity on these experimental parameters is heuristically captured in a single power law. Further, the late-time mass distributions obtained are shown to be governed by a single parameter. These studies are performed under conditions relevant for plasma light sources for extreme-ultraviolet nanolithography.
\end{abstract}

DOI: 10.1103/PhysRevApplied.10.054005

\section{INTRODUCTION}

Intense, short-pulse laser radiation can produce strong shock waves in liquids, leading in some spectacular cases to explosive cavitation and violent spallation of the material [1-5]. Such dramatic physical phenomena can readily find applications, a very recent example being in the field of nanolithography where microdroplets of liquid tin are used to create extreme-ultraviolet (EUV) light [6-8]. These tin droplets, typically several tens of micrometers in diameter, serve as targets for a highenergy, nanosecond-pulse laser, creating a laser-produced plasma. Line emission from highly charged tin ions in the laser-produced plasma provides the required EUV light. Currently, a dual-laser-pulse sequence is used [8]. In a first step, a nanosecond-laser prepulse is used to carefully shape the droplet into a thin sheet that is considered to be advantageous for EUV production with a second, much-more-energetic, main pulse. Recent developments $[8,9]$, however, produced tentative but tantalizing evidence

\footnotetext{
*versolato@arcnl.nl
}

Published by the American Physical Society under the terms of the Creative Commons Attribution 4.0 International license. Further distribution of this work must maintain attribution to the author(s) and the published article's title, journal citation, and DOI. for significantly improved source performance when the nanosecond prepulse was replaced with a picosecond prepulse to produce shock-wave-induced explosive fragmentation. Although some notable progress was made very recently $[3-5,10]$, this process requires further investigation.

This paper advances the understanding of the aforementioned systems by providing an experimental and theoretical study of the late-time dynamics of the deformation of free-falling tin microdroplets. The initially spherical droplets are subjected to strong shock waves generated by picosecond-laser-pulse impact, giving rise to cavitation $[3-5,10]$. This centralized cavitation explosively propels the liquid to very high (approximately $100 \mathrm{~m} / \mathrm{s}$ ) radial velocities, producing a rapidly thinning liquid tin shell (see Fig. 1). The initial spherical symmetry of the system is broken when the intensity of the shock wave exceeds a certain threshold, and dramatic spallation is observed on the side of the droplet facing away from the laser-impact zone. We focus our studies on the rich physics of the dynamics set in motion by the central cavitation.

We develop a model description for the time evolution of a stretching spherical shell, including the onset of fragmentation. Using stroboscopic shadowgraphic imaging, we experimentally validate this model over a wide range of laser pulse energies, $15 \mathrm{ps}$ in duration, and for two droplet sizes. The dependence of the initial expansion 


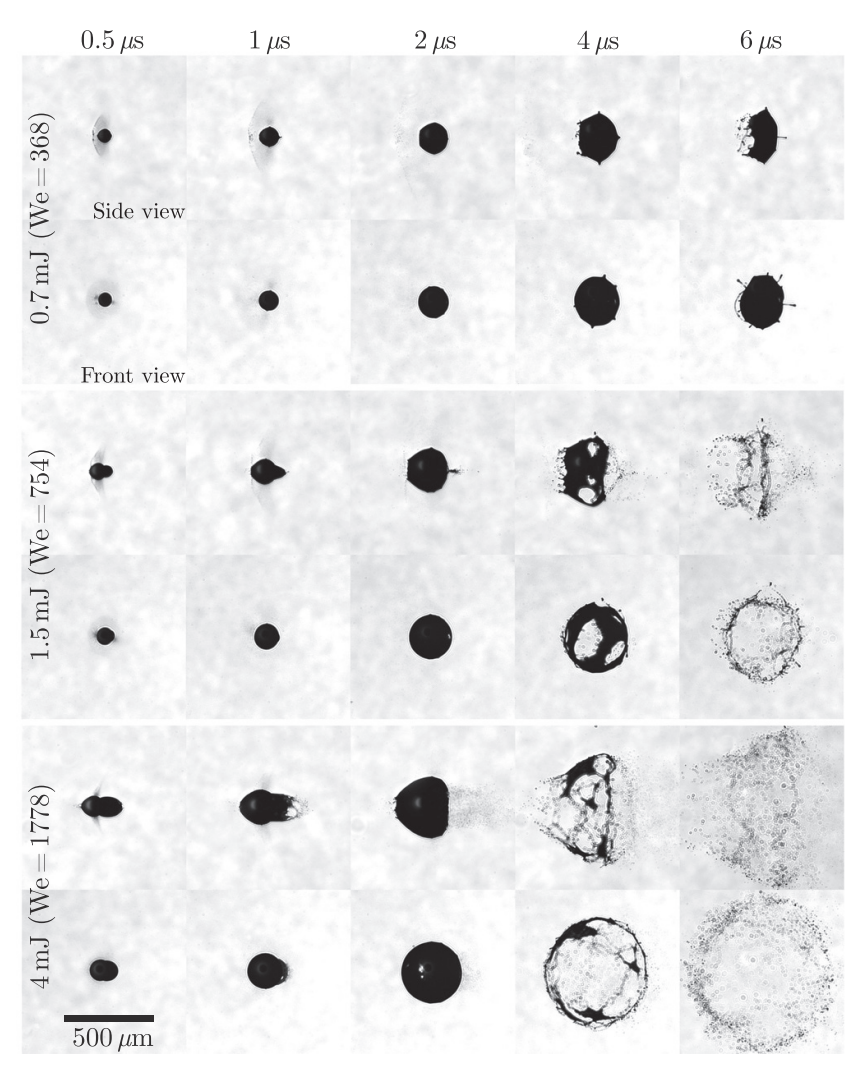

FIG. 1. Stroboscopic shadowgraph images of expanding tin microdroplets $(23-\mu \mathrm{m}$ initial radius) taken at different time delays for three different laser-pulse energies (or Weber numbers, see Sec. III A) at a pulse length of 15 ps, as seen from two viewing angles $\left(90^{\circ}\right.$ side view and $30^{\circ}$ front view). Laser impacts from the left; images are cropped and centered individually to improve visibility. A $500-\mu \mathrm{m}$ scale bar is provided in the left-lower corner.

velocity on these experimental parameters is heuristically captured in a single power law. The late-time mass distributions, experimentally obtained from front-view shadowgraphy, are furthermore shown to be governed by a single parameter.

\section{EXPERIMENTAL METHODS}

Our experimental setup has previously been described in detail [11]. For clarity, the most-important characteristics, as well as experimental upgrades, are discussed here. A droplet generator is operated in a vacuum chamber $\left(10^{-7} \mathrm{mbar}\right)$ and held at constant temperature of $260^{\circ} \mathrm{C}$, well above the melting point of tin. The nozzle produces an on-axis multikilohertz train of 15 - or $23-\mu$ m-radius droplets of $99.995 \%$ purity tin, with density $\rho=7 \mathrm{~g} / \mathrm{cm}^{3}$ and surface tension $\sigma=0.532 \mathrm{~N} / \mathrm{m}$. The droplets relax to a spherical shape before they pass through a horizontal light sheet produced by a helium-neon laser. The light scattered by the droplets is detected by a photomultiplier tube, whose signal is reduced in frequency to $10 \mathrm{~Hz}$ to trigger a Nd:YAG laser system. This laser system produces tunable picosecond pulses of 1064-nm-wavelength light, as described in more detail in Ref. [12]. The laserpulse energy is varied between 0.5 and $5 \mathrm{~mJ}$ by use of a suitable combination of a waveplate and polarizer; the pulse length is kept constant at approximately $15 \mathrm{ps}$ for the experiments described here. The laser beam is focused to a Gaussian spot with a full-width-at-half-maximum diameter of $100 \mu \mathrm{m}$. To maintain cylindrical symmetry, the laser light has circular polarization. An accurately timed laser pulse provides a radially centered interaction with a falling droplet that occurs in a reproducible manner. Only a small fraction of the droplets interact with a laser pulse due to the mismatch in repetition rates of the droplet generator (multikilohertz) and the laser $(10 \mathrm{~Hz})$.

Two shadowgraph imaging systems capture the dynamics of the expanding droplets. These systems are based on a single pulse from a broadband $560 \pm 2 \mathrm{~nm}$ wavelength 5-ns-pulse dye laser. Combined with two long-distance microscopes coupled with CCD cameras, this laser source provides backlighting that is carefully rendered incoherent both temporally and spatially, with $4-\mu \mathrm{m}$ resolution. One of these microscopes is aligned orthogonally to the laser beam to obtain side-view images; the other one is at $30^{\circ}$ to the beam direction for a (tilted) front view. Both microscopes are equipped with bandpass filters to suppress the plasma radiation. The images obtained are used to track the size, shape, and velocity of the droplet expansion with use of an image-processing algorithm. Stroboscopic time series of different droplets are constructed by our triggering the shadowgraphy systems once per drive-laser shot with increasing delay, typically with time steps of 50-100 ns [13].

\section{RESULTS AND INTERPRETATION}

The response of a tin droplet to laser-pulse impact is shown in Fig. 1 for three laser-pulse energies. A qualitative description of the relevant physical processes leading to cavitation and spallation was recently given in Ref. [4]. We summarize the crucial steps in the following, combining this with our experimental observations. The laser-pulse impact ablates a thin (less-than-1- $\mu \mathrm{m}$ ) layer of tin. At the lower pulse energies (see the $0.7-$ and $1.5-\mathrm{mJ}$ cases presented in Fig. 1), this ablated mass is clearly visible and is seen to move away from the droplet in the direction opposite the laser light. The remarkably sharp outer boundary of this ablated mass may be explained by the existence of an inhomogeneous two-phase, gas-liquid mixture of low average density but approaching liquid density in the vicinity of the ablation front $[14,15]$.

The ablation pulse gives rise to a pressure wave starting from the laser-impacted region of the droplet. This pressure is applied on a short, picosecond timescale that is 
several orders of magnitude shorter than the timescale on which pressure waves travel through the droplet.

As a consequence, the liquid is locally compressed and pressure waves travel through the droplet [16]. Converging pressure "shock" waves are superimposed in the center of the droplet, where they cause tensile stress. Once this tensile stress is above the yield strength of the liquid, it ruptures and a thin shell is formed. Theoretical modeling [4] has shown that there is high sensitivity of both the rate of expansion and the morphology of the deformed droplet to the details of the, poorly known, metastable equation of state (EOS) of tin in the region of the liquidvapor phase transition and to the parameters of the critical point. Thus, precise theoretical predictions of the postcavitation dynamics of liquid tin microdroplets cannot yet be made, and instead, measurements as presented in this work may provide a sensitive instrument for probing the EOS of liquid metals [4].

After passing through the droplet center, the shock wave reaches the rear side of the droplet (i.e., the side facing away from the laser) and is reflected from this free interface, where it can again rupture the liquid and give rise to spallation that rapidly propels, at several hundred meters per second, a small mass fraction of the droplet along the laser-beam propagation direction (see Fig. 1) [4,5]. The fluid-dynamic description of this spallation is very rich and is left for future work. However, it is noteworthy that there is a regime where strong spallation occurs, but this is mitigated under capillary action (compare the 1- and 2- $\mu \mathrm{s}$ shadowgraphs for the 1.5-mJ case in Fig. 1).

The higher-energy cases in Fig. 1 also hint at the existence of a hole on the side of the droplet facing the laser, which may possibly be attributed to ablation-thinning of the droplet shell. In combination with the spallation, a "tunnel" is thus created (see the front-view shadowgraphs in Fig. 1), and may later form a doughnut-type mass distribution. Another scenario, when fragmentation following the collapse of the shell results in high-speed jetting, corresponding to the lowest laser-pulse energies, is reported in Refs. $[10,17,18]$.

In the following, we focus on the late-time dynamics set in motion by the central cavitation. Firstly, a basic model for the time evolution of the intact liquid shell is presented. Secondly, we study the timescale on which first holes close to the equator of this shell become visible, after which full fragmentation of the shell rapidly sets in. This results, eventually, in a late-time mass distribution, the understanding of which is of particular relevance for producing EUV radiation with high conversion efficiencies.

\section{A. Time evolution of the shell}

The shadowgraphs such as those presented in Fig. 1 clearly show a cylindrically symmetric expanding shell, the radius $R(t)$ of which is tracked for each time step by our measuring the maximal transverse (vertical in Fig. 1) size of this feature from the side-view shadowgraphs. By fitting a linear function to the first few $R(t)$ data points after the laser impact, we obtain values for the initial droplet expansion velocity $\dot{R}(t=0)$. Figure 2(a) shows a monotonic increase of this radial expansion velocity $\dot{R}(t=0)$ as a function of laser-pulse energy. Using a heuristic argument, we collapse all data obtained onto a single curve, scaling the velocities obtained with the initial droplet radius $R_{0}$ [see Fig. 2(b)]. To arrive at this scaling, we hypothesize that the magnitude of the induced shock wave, and its related cavitation event, scales with the laserfacing surface area, proportional to $R_{0}^{2}$ (the droplet is much smaller than the laser-spot size). Meanwhile, the expansion is impeded by the droplet mass proportionally to $R_{0}^{-3}$. By ignoring the possible differences in the dissipation of the shock wave, one thus arrives at the aforementioned simple scaling $\dot{R}(t=0) \propto R_{0}^{-1}$. Ablation-induced momentum exerted on the droplet, leading to propulsion, is expected also to scale with absorbed energy, again proportional to $R_{0}^{2}[11,19]$, counteracted by the droplet mass as proportional to $R_{0}^{3}$, leading to an identical scaling law. However, in contrast to the nanosecond-laser-pulse impact studied in Refs. [11,19], the resulting droplet propulsion is limited here. As seen from Fig. 2(b), such a rescaling causes all data to fall on a single curve that appears

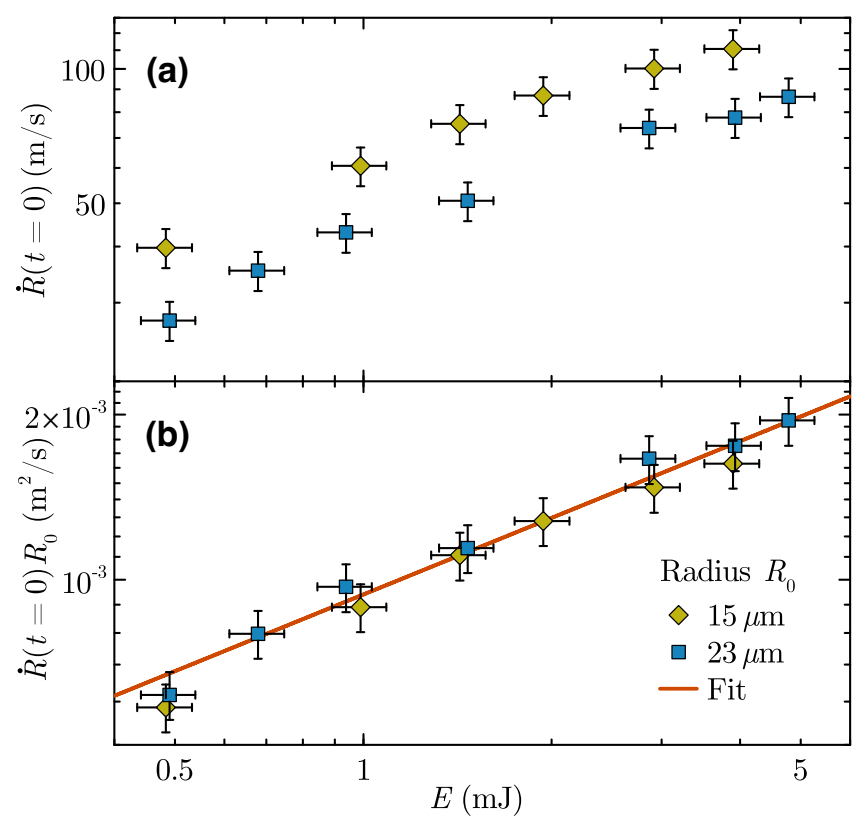

FIG. 2. (a) Initial radial expansion velocity $\dot{R}(t=0)$ of tin microdroplets as a function of total laser-pulse energy $E$. Yellow diamonds are for $R_{0}=15 \mu \mathrm{m}$ and blue squares are for $R_{0}=23 \mu \mathrm{m}$. The error bars represent $10 \%$ uncertainty on the velocity measurements and $20 \%$ uncertainty on the energy measurements. (b) The same data, rescaled by multiplication of by the initial droplet radius $R_{0}$. The solid red line is obtained by the fitting of a power-law function to the concatenated data. 
to be represented quite well by a power-law function $\dot{R}(t=0) R_{0}=A \times E^{\alpha}$ with fit parameters $A=9.4 \pm$ $1.4 \mathrm{~m}^{2} / \mathrm{s}$ and $\alpha=0.46 \pm 0.02$. Although such powerlaw behavior is well established in the relevant literature on ablation [20-22], these fit parameters cannot be straightforwardly predicted from available theory, given their sensitivity to the EOS and the details of laser-matter interaction.

To describe the time evolution of the liquid droplets in Fig. 1, we hypothesize that a small (micrometer-sized), high-pressure (kilobar-level) cavitation bubble expands the liquid quickly into a thin shell. Thus, a spherically symmetric shell expands at a certain initial velocity $\dot{R}(t=0)$, with the cavitation pressure having done its thermodynamic work effectively at time zero. Given that the droplet expands into the vacuum, the only limitation to its expansion is surface tension.

The liquid tin shell has thickness $h(t)$, which decreases quadratically with time as mass conservation implies, in the limit of a thin shell $[R(t) \gg h(t)]$, that $h(t)=R_{0}^{3} /\left(3 R(t)^{2}\right) \approx R_{0}^{3} /\left(3 t^{2} \dot{R}(t=0)^{2}\right)$. The expansion of the shell can be described by the Rayleigh-Plesset equation, which in the limit of a thin shell reads [23] $p(R-h)-p(R) \approx \rho h \ddot{R}$, where $p$ is pressure. Imposing dynamic boundary conditions at the two liquid-vapor interfaces $p(R-h)$ and $p(R)$, and noting that the only force acting on the shell is the Laplace pressure, the net value of which is given by $p=4 \sigma / R(t)$ (in contrast to Ref. [23], where this contribution was negligible), we find

$$
\ddot{R}(t)=\frac{-p}{\rho h(t)}=-\frac{4 \sigma}{\rho h(t) R(t)}=-\frac{12 \sigma R(t)}{\rho R_{0}^{3}} .
$$

From the above expression, using the boundary conditions of a given $\dot{R}(t=0)$ and initial radius $R_{0}$, we obtain

$$
\frac{R(t)}{R_{0}}=\cos \left(\pi t / \tau_{c}\right)+\sqrt{\frac{\mathrm{We}}{12}} \sin \left(\pi t / \tau_{c}\right),
$$

introducing the capillary timescale here as $\tau_{c}=\pi \sqrt{\rho R_{0}^{3} / 12 \sigma}$ and the relevant Weber number as $\mathrm{We}=\rho R_{0} \dot{R}(t=0)^{2} / \sigma$. The above solution is identical in form to that obtained previously for a disk-type expansion of a droplet following nanosecond-laser-pulse impact $[11,24]$.

Next we apply the theory developed above with zero fit parameters taking as an input the experimental values for $\dot{R}(t=0)$. The results are presented in Fig. 3(b), where the experimentally obtained radius $R(t)$ [see Fig. 3(a)] is rendered dimensionless by division by $R_{0}$ (and subtraction of the unity-value offset), and is plotted as a function of dimensionless time $t / \tau_{c}$. For $R_{0}=15$ the capillary time is approximately $6 \mu \mathrm{s}$; for $R_{0}=23 \mu \mathrm{m}$ it is approximately $11 \mu \mathrm{s}$. Given the simplicity of our arguments, we
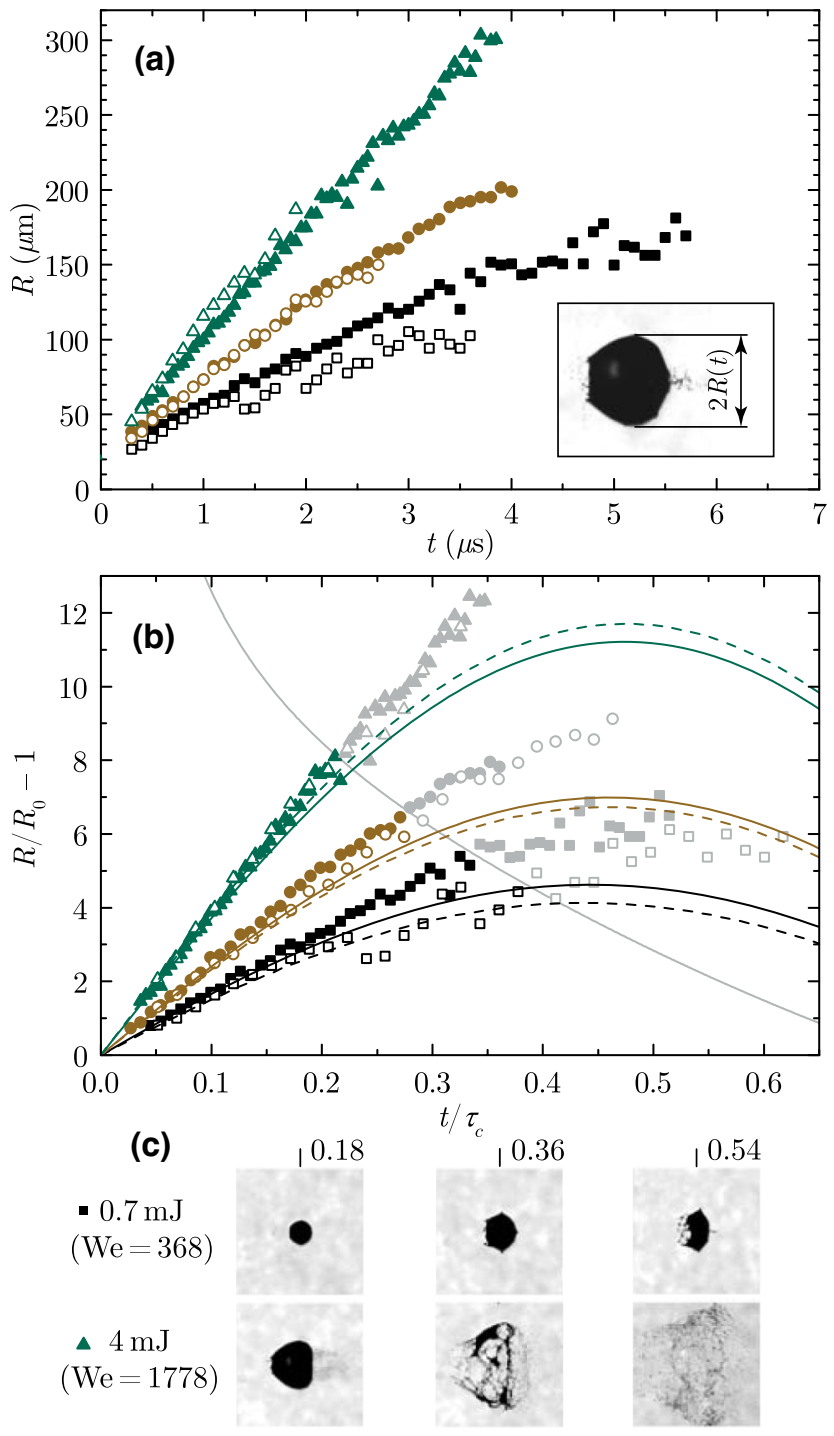

FIG. 3. (a) Radius $R(t)$ of shell driven by cavitation in $R_{0}=$ $15 \mu \mathrm{m}$ droplets (open symbols) and $R_{0}=23 \mu \mathrm{m}$ droplets (colored symbols) for similar Weber numbers. Open squares, We = 304; black squares, $\mathrm{We}=368$; open circles, We $=705$; brown circles, $\mathrm{We}=754$; open triangles, $\mathrm{We}=1923$; green triangles, $\mathrm{We}=1778$. The inset $\left(500 \times 700 \mu \mathrm{m}^{2}\right)$ shows a side-view image of the We $=754$ case at $2.5 \mu \mathrm{s}$. (b) Rescaled, dimensionless radius as a function of dimensionless time $t / \tau_{c}$. The solid curves represent the model predictions [see Eq. (2)] for the 23- $\mu \mathrm{m}$-initial-radius droplet; the dashed curves show the same but for the smaller, $15-\mu \mathrm{m}$ droplet. The solid gray line depicts the destabilization radius $R\left(t_{*}\right)$ as obtained from the fit of Eq. (3) to the data (see Fig. 4), applied to Eq. (2). The data are grayed out beyond this line. (c) Sequence of side-view shadowgraphy images taken from Fig. 1 for $\mathrm{We}=368$ and $\mathrm{We}=1778$ for $R_{0}=23 \mu \mathrm{m}$ droplets, with indication of the corresponding dimensionless time $t / \tau_{c}$.

find excellent agreement between the model and experiment. The observed late-time "overshoot" that is apparent in the experimental data shown, with theory predicting a more rapid retraction of the shell, can be understood by 
consideration of the process of hole formation and ensuing fragmentation, which strongly reduces the restoring forces.

\section{B. Hole opening time}

The expanding shell is subjected to Rayleigh-Taylor instabilities (RTIs), here as instabilities of the liquid-vapor interface driven by the radial acceleration and deceleration of the liquid sheet [23]. Surface tension provides mode selection of the RTI [23,25-27]. This phenomenon will lead to hole formation, after which rapid hole opening and merging will lead to full fragmentation of the shell. In our system, there are two acceleration mechanisms that act along the surface normal and that thus will contribute to RTI growth. Firstly, the liquid is strongly accelerated by the cavitation pressure. Secondly, after the initial fast expansion, the shell much more slowly decelerates under the influence of surface tension. Holes will form at the shell piercing time $t_{*}$, when the size of a growing instability is of the order of the shell thickness [23]. In the available literature (e.g., see Refs. $[23,27])$, quite generally a scaling relation is found of the type

$$
\frac{t_{*}}{\tau_{c}} \propto \mathrm{We}^{-\beta},
$$

resulting in a rough scaling for the corresponding destabilization radius [23]

$$
\frac{R\left(t_{*}\right)}{R_{0}}-1 \propto \mathrm{We}^{1 / 2-\beta} .
$$

Here we have, for simplicity, linearized the expansion rate to a constant $\dot{R} \propto \mathrm{We}^{1 / 2}$ and in Eq. (3) dropped the usual weak scaling term $\left(\eta_{0} / R_{0}\right)^{\beta}$, where $\eta_{0}$ is the initial amplitude of RTI. The positive power $\beta$ is typically smaller than unity $[23,27,28]$. Obtaining a theoretical value for this power is complicated because of the two competing mechanisms driving RTI. We take instead the rather general form of Eq. (3) and let the experiment provide the relevant value for $\beta$ and for the proportionality constant. In the experiments, a hole close to the equator of the shell becomes clearly visible in side-view shadowgraphy only when another hole simultaneously appears on the opposite side. This naturally introduces a slight detection bias. However, it is possible to reliably and reproducibly obtain an estimate for $t_{*}$ based on optical inspection of the experimental data supported by the fact that for times $t>t_{*}$ (where we take 100-ns time steps) the shell becomes permeated with holes. We observe a monotonically decreasing value for $t_{*}$ with increasing Weber number as expected from Eq. (3). Furthermore, the absolute time of breakup $t_{*}$ is seen to decrease with droplet size for similar Weber numbers.

Rescaling $t_{*} \rightarrow t_{*} / \tau_{c}$ in Fig. 4 indeed collapses the data for the two different droplet sizes onto a single curve. The resulting fit values of the power law (3) are given by

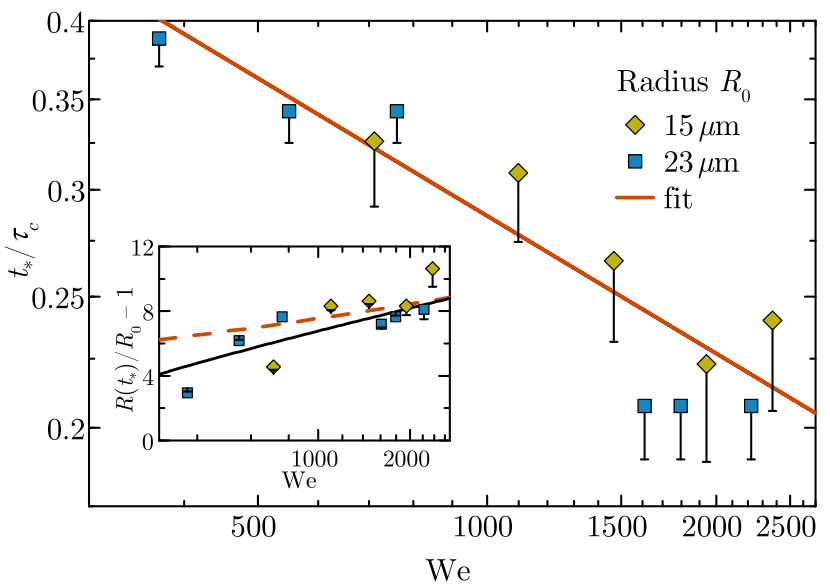

FIG. 4. Dimensionless time of first hole opening $t_{*} / \tau_{c}$ as a function of Weber number for two droplet sizes: $15-\mu \mathrm{m}$ initial radius (yellow diamonds) and $23-\mu \mathrm{m}$ initial radius (blue squares). For $\mathrm{We} \lesssim 300$, the shell collapses before holes are apparent. Error bars are two time steps toward shorter values. The solid red line represents the best fit of a power law to the concatenated data. The inset depicts the dimensionless radius of the shell at the moment of hole opening. The solid black line shows the destabilization radius as obtained from insertion of Eq. (3) into Eq. (2). The dashed red line is based on Eq. (4) taking $\beta=0.34$ (see the main text).

$\beta=0.34(4)$ and 3.0(8) for the proportionality constant. The apparent similarity of the hole opening times for the larger droplet for We $>1500$ should be considered to be purely coincidental, linked to the finite accuracy of the optical inspection method of a selected data set with limited temporal resolution. As we clearly find $\beta<1 / 2$, we can now immediately conclude from Eq. (4) that the destabilization radius $R\left(t_{*}\right)$ increases with Weber number, and thus with laser energy. The fit values obtained will serve as input for predicting the late-time mass distribution produced by the laser impact.

\section{Late-time mass distribution}

Having achieved good agreement between experiment and the model, we now summarize our findings in Fig. 5 to facilitate a more direct, industrial application of our findings. The maximum amplitude of an intact retracting shell is given by our maximizing Eq. (2). The resulting dashed black curve in Fig. 5 is close to the well-known scaling as approximately $\sqrt{\mathrm{We}}$ (as pointed out in Ref. [29]). The maximum attainable shell size without any holes (i.e., the destabilization radius) is obtained by insertion of Eq. (3) into Eq. (2) as in Fig. 3. The result is shown as a solid black line in Fig. 5. Once this line is crossed in the expansion phase, the rapid breakup and fragmentation of the shell, associated with large Weber numbers here, precludes capillary collapse, and the droplet target fragments 


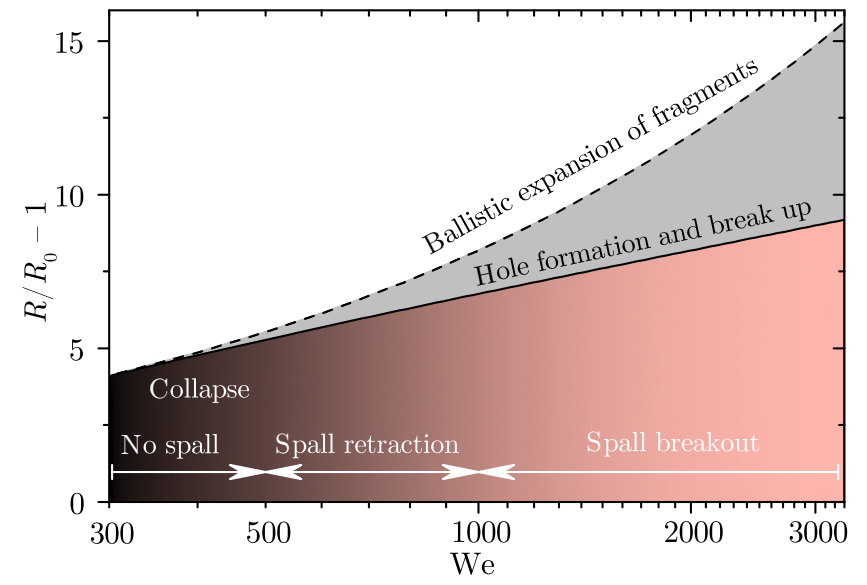

FIG. 5. Phase diagram depicting the maximum obtainable radius as a function of Weber number. White arrows illustrate the various spallation regimes (see the main text). The dashed line is obtained by maximization of Eq. (2). The solid line shows the destabilization radius as obtained from insertion of Eq. (3) into Eq. (2); the area under this line is obtainable without holes occurring.

ballistically expand. For the low Weber numbers shown, the shell collapses and jetting may ensue [10].

In Fig. 5 we also indicate ranges of Weber numbers that we associate with different spallation regimes. For Weber numbers below approximately 500 , no spallation is visible at all, for $500 \lesssim \mathrm{We} \lesssim 1000$, the spall is nearly fully retracted due to surface tension, and for We $\gtrsim 1000$, there is a rather abrupt and violent breakout of the spall. For We $\gtrsim 1000$, we find that hole formation in the "tunnel" wall leads very quickly to complete breakup of the shell, where the fragments continue on their ballistic trajectories with radial velocities close to the initial $\dot{R}(t=0)$.

From an application perspective, it is interesting to study the "final" late-time fragment mass distribution that might serve as a target for a main laser pulse in an actual industrial EUV source based on tin plasma. In the following analysis, we present some brief guidelines based on the data presented here but keep the full analysis of the relevant fragmentation process (including fragment size distributions) for later, dedicated work. We find that the final mass distribution changes very dramatically over a relatively small range of Weber numbers as can be seen from the shadowgraphs presented in Fig. 6(a) as well as from their angularly averaged, radial projections shown in Fig. 6(b). These projections can loosely be interpreted as a column mass density distribution along the line of sight of the backlight illumination. The projections are not corrected for the small parallax angle or for the limited depth of focus. Still, we expect to track a large fraction of the total droplet mass. Similar mass distributions are found for $15-$ and $23-\mu \mathrm{m}$ droplets at comparable Weber numbers (see Fig. 6).
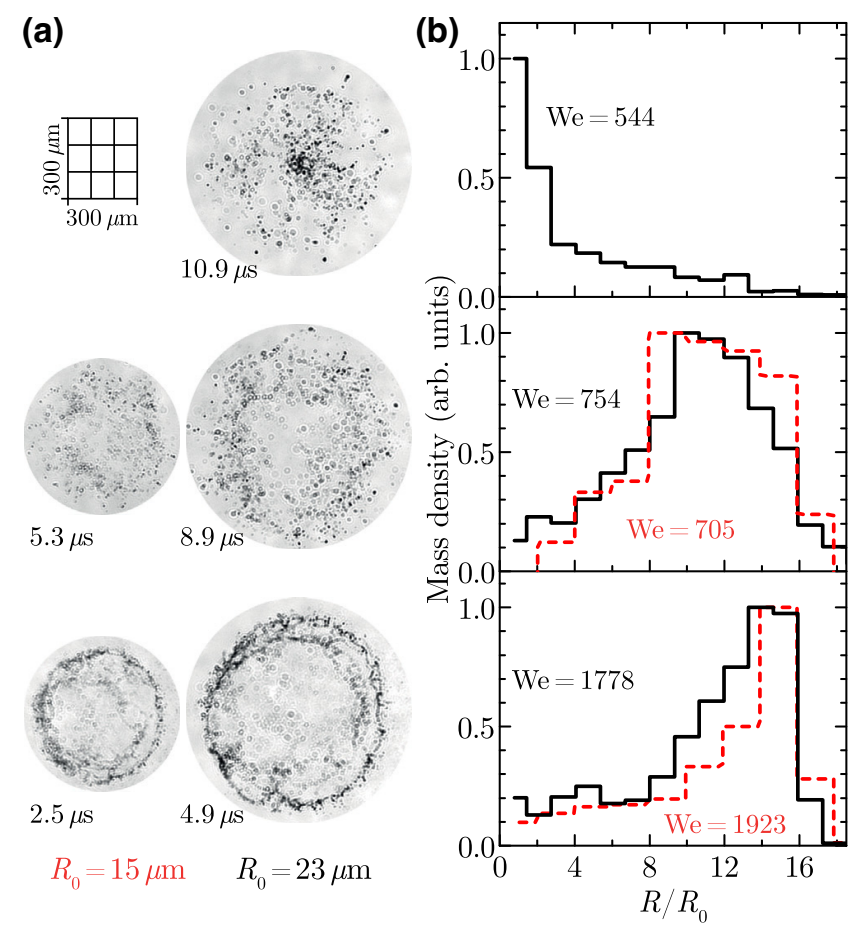

FIG. 6. (a) Late-time front-view $\left(30^{\circ}\right)$ shadowgraphs of expanded and deformed droplets of different droplet sizes representing cases of similar Weber numbers. The rectangular scale grid in the top-left corner visualizes the effect of the $30^{\circ}$ parallax angle. (b) Inverted, angularly averaged, radial projections of the obtained grayscale of the corresponding images from (a), which give a qualitative measure of the radial column mass density distribution. The dashed red line corresponds to the data from $R_{0}=15 \mu \mathrm{m}$ droplets; the solid black line is for $R_{0}=23 \mu \mathrm{m}$.

For $\mathrm{We}=544$ we observe that the mass distribution has a maximum in the center of the image, as is to be expected from a collapse. For just very slightly larger Weber numbers, we find instead a toroidal profile that becomes more and more pronounced for larger Weber numbers. We attribute this observation to the RTI-driven breakup of the "tunnel" wall (thus preventing collapse) as well as a pronounced spallation that modifies the mass distribution, as was also noted in Ref. [4].

The sensitivity of the final state mass distribution to the Weber number established and quantified here can readily find application. As an example, we note that a typical target size used in industry is on the order of a few hundred micrometers [8], several microseconds after prepulse impact. In our experiments, a relevant approximately-450$\mu \mathrm{m}$-diameter target is, for example, obtained from an $R_{0}=$ $15 \mu \mathrm{m}$ initial droplet size after expansion for $2.5 \mu \mathrm{s}$; see Fig. 6(a) for $\mathrm{We}=1923$. In this case, the main pulse would not find a significant mass fraction in its focus. Thus, it may well be opportune to choose a lower Weber number, by tuning down the laser energy, and obtain a more homogeneous mass distribution, which could increase the conversion efficiency of drive-laser light into useful EUV 
radiation by, for example, increasing the absorption of drive-laser light.

\section{CONCLUSIONS}

We present an analysis of the cavitation-driven expansion dynamics of liquid tin microdroplets that is set in motion by the ablative impact of a 15-ps laser pulse. High-resolution stroboscopic shadowgraphy of the expanding tin shells is combined with an intuitive fluiddynamic model that includes the time and size at which the onset of fragmentation becomes apparent. This model will aid follow-up studies of the fragmentation pathways. Good agreement between model and experimental data is found for two different droplet sizes over a wide range of laserpulse energies. The dependence of the initial expansion velocity of the liquid shell on these experimental parameters is heuristically captured, for application purposes, in a single power law. A summary phase diagram of the expansion dynamics is presented. It covers regimes with and without spallation, as well as a transition regime where the spalled material is retracted under surface tension. This transition regime enables one to find conditions for a maximum shell expansion velocity with strongly suppressed forward-moving debris. This phase diagram facilitates a more direct, industrial application of our findings.

Further, the experimentally obtained late-time mass distributions are shown to be governed by a single parameter, the Weber number. These studies are of particular relevance for plasma sources of extreme-ultraviolet light for nanolithography. In such plasma sources, the tin mass distributions obtained upon cavitation-driven shell fragmentation, as studied in this work, are shown to be promising targets for efficient laser coupling [8,9].

\section{ACKNOWLEDGMENTS}

We thank A.L. Klein and M.M. Basko for insightful discussions. This work was conducted at the Advanced Research Center for Nanolithography, a public-private partnership between the University of Amsterdam, Vrije Universiteit Amsterdam, the Netherlands Organization for Scientific Research (NWO), and the semiconductorequipment manufacturer ASML.

[1] C. A. Stan, D. Milathianaki, H. Laksmono, R. G. Sierra, T. A. McQueen, M. Messerschmidt, G. J. Williams, J. E. Koglin, T. J. Lane, M. J. Hayes, S. A. H. Guillet, M. Liang, A. L. Aquila, P. R. Willmott, J. S. Robinson, K. L. Gumerlock, S. Botha, K. Nass, I. Schlichting, R. L. Shoeman, H. A. Stone, and S. Boutet, Liquid explosions induced by x-ray laser pulses, Nat. Phys. 12, 966 (2016).

[2] C. A. Stan, P. R. Willmott, H. A. Stone, J. E. Koglin, M. Liang, A. L. Aquila, J. S. Robinson, K. L. Gumerlock, G.
Blaj, R. G. Sierra, S. Boutet, S. A. H. Guillet, R. H. Curtis, S. L. Vetter, H. Loos, J. L. Turner, and F.-J. Decker, Negative pressures and spallation in water drops subjected to nanosecond shock waves, J. Phys. Chem. Lett. 7, 2055 (2016).

[3] A. Y. Vinokhodov, K. N. Koshelev, V. M. Krivtsun, M. S. Krivokorytov, Y. V. Sidelnikov, V. V. Medvedev, V. O. Kompanets, A. A. Melnikov, and S. V. Chekalin, Formation of a fine-dispersed liquid-metal target under the action of femto- and picosecond laser pulses for a laser-plasma radiation source in the extreme ultraviolet range, Quantum Electron. 46, 23 (2016).

[4] M. M. Basko, M. S. Krivokorytov, A. Y. Vinokhodov, Y. V. Sidelnikov, V. M. Krivtsun, V. V. Medvedev, D. A. Kim, V. O. Kompanets, A. A. Lash, and K. N. Koshelev, Fragmentation dynamics of liquid-metal droplets under ultra-short laser pulses, Laser Phys. Lett. 14, 036001 (2017).

[5] M. S. Krivokorytov, A. Y. Vinokhodov, Y. V. Sidelnikov, V. M. Krivtsun, V. O. Kompanets, A. A. Lash, K. N. Koshelev, and V. V. Medvedev, Cavitation and spallation in liquid metal droplets produced by subpicosecond pulsed laser radiation, Phys. Rev. E 95, 031101 (2017).

[6] V. Y. Banine, K. N. Koshelev, and G. H. P. M. Swinkels, Physical processes in EUV sources for microlithography, J. Phys. D: Appl. Phys. 44, 253001 (2011).

[7] A. Pirati, J. van Schoot, K. Troost, R. van Ballegoij, P. Krabbendam, J. Stoeldraijer, E. Loopstra, J. Benschop, J. Finders, H. Meiling, E. van Setten, N. Mika, J. Dredonx, U. Stamm, B. Kneer, B. Thuering, W. Kaiser, T. Heil, and S. Migura, The future of EUV lithography: Enabling Moore's Law in the next decade, Proc. SPIE 10143, 10143-10143-16 (2017).

[8] I. Fomenkov, D. Brandt, A. Ershov, A. Schafgans, Y. Tao, G. Vaschenko, S. Rokitski, M. Kats, M. Vargas, M. Purvis, R. Rafac, B. La Fontaine, S. De Dea, A. LaForge, J. Stewart, S. Chang, M. Graham, D. Riggs, T. Taylor, M. Abraham, and D. Brown, Light sources for highvolume manufacturing EUV lithography: Technology, performance, and power scaling, Adv. Opt. Technol. 6, 173 (2017).

[9] Y. Kawasuji, K. M. Nowak, T. Hori, T. Okamoto, H. Tanaka, Y. Watanabe, T. Abe, T. Kodama, H. Nakarai, T. Yamazaki, S. Okazaki, T. Saitou, H. Mizoguchi, and Y. Shiraishi, Key components technology update of the $250 \mathrm{~W}$ high-power LPP-EUV light source, Proc. SPIE 10143, 10143-10143-11 (2017).

[10] M. S. Krivokorytov, Q. Zeng, B. V. Lakatosh, A. Y. Vinokhodov, Y. V. Sidelnikov, V. O. Kompanets, V. M. Krivtsun, K. N. Koshelev, C.-D. Ohl, and V. V. Medvedev, Shaping and controlled fragmentation of liquid metal droplets through cavitation, Sci. Rep. 8, 597 (2018).

[11] D. Kurilovich, A. L. Klein, F. Torretti, A. Lassise, R. Hoekstra, W. Ubachs, H. Gelderblom, and O. O. Versolato, Plasma propulsion of a metallic microdroplet and its deformation upon laser impact, Phys. Rev. Appl. 6, 014018 (2016).

[12] A. S. Stodolna, T. de Faria Pinto, F. Ali, A. Bayerle, D. Kurilovich, J. Mathijssen, R. Hoekstra, O. O. Versolato, K. S. E. Eikema, and S. Witte, Controlling ion kinetic energy distributions in laser produced plasma sources by 
means of a picosecond pulse pair, J. Appl. Phys. 124, 053303 (2018).

[13] See Supplemental Material at http://link.aps.org/supple mental/10.1103/PhysRevApplied.10.054005 for stroboscopic shadowgraphy movies.

[14] K. Sokolowski-Tinten, J. Bialkowski, A. Cavalleri, D. von derLinde, A. Oparin, J. Meyer-ter-Vehn, and S. I. Anisimov, Transient States of Matter During Short Pulse Laser Ablation, Phys. Rev. Lett. 81, 224 (1998).

[15] D. von der Linde and K. Sokolowski-Tinten, The physical mechanisms of short-pulse laser ablation, Appl. Surf. Sci. 154-155, 1 (2000).

[16] S. A. Reijers, J. H. Snoeijer, and H. Gelderblom, Droplet deformation by short laser-induced pressure pulses, J. Fluid Mech. 828, 374 (2017).

[17] S. T. Thoroddsen, K. Takehara, T. Etoh, and C.-D. Ohl, Spray and microjets produced by focusing a laser pulse into a hemispherical drop, Phys. Fluids 21, 112101 (2009).

[18] S. R. Gonzalez-Avila, E. Klaseboer, B. C. Khoo, and C.-D. Ohl, Cavitation bubble dynamics in a liquid gap of variable height, J. Fluid Mech. 682, 241 (2011).

[19] D. Kurilovich, M. M. Basko, D. A. Kim, F. Torretti, R. Schupp, J. C. Visschers, J. Scheers, R. Hoekstra, W. Ubachs, and O. O. Versolato, Power-law scaling of plasma pressure on laser-ablated tin microdroplets, Phys. Plasmas 25, 012709 (2018).

[20] K. Eidmann, F. Amiranoff, R. Fedosejevs, A. G. M. Maaswinkel, R. Petsch, R. Sigel, G. Spindler, Y.-1. Teng,
G. Tsakiris, and S. Witkowski, Interaction of 1.3-m laser radiation with thin foil targets, Phys. Rev. A 30, 2568 (1984).

[21] F. Dahmani, Experimental scaling laws for mass-ablation rate, ablation pressure in planar laser-produced plasmas with laser intensity, laser wavelength, and target atomic number, J. Appl. Phys. 74, 622 (1993).

[22] K. Eidmann, J. Meyer-ter-Vehn, T. Schlegel, and S. Hüller, Hydrodynamic simulation of subpicosecond laser interaction with solid-density matter, Phys. Rev. E 62, 1202 (2000).

[23] A. Vledouts, J. Quinard, N. Vandenberghe, and E. Villermaux, Explosive fragmentation of liquid shells, J. Fluid Mech. 788, 246 (2016).

[24] H. Gelderblom, H. Lhuissier, A. L. Klein, W. Bouwhuis, D. Lohse, E. Villermaux, and J. H. Snoeijer, Drop deformation by laser-pulse impact, J. Fluid Mech. 794, 676 (2016).

[25] J. B. Keller and I. Kolodner, Instability of liquid surfaces and the formation of drops, J. Appl. Phys. 25, 918 (1954).

[26] S. Chandrasekhar, Hydrodynamic and Hydromagnetic Stability (Oxford University Press, Oxford, U.K., 1961).

[27] N. Bremond and E. Villermaux, Bursting thin liquid films, J. Fluid Mech. 524, 121 (2005).

[28] A. Klein, Ph.D. thesis, University of Twente, 2017.

[29] A. L. Klein, W. Bouwhuis, C. W. Visser, H. Lhuissier, C. Sun, J. H. Snoeijer, E. Villermaux, D. Lohse, and H. Gelderblom, Drop shaping by laser-pulse impact, Phys. Rev. Appl. 3, 044018 (2015). 